\title{
Primary Age-Related Tauopathy: An Elderly Brain Pathology Frequently Encountered during Autopsy
}

Daru Kim · Hyung-Seok Kim ${ }^{1}$ Seong-Min Choi ${ }^{2}$. Byeong C. Kim ${ }^{2}$ Min-Cheol Lee · Kyung-Hwa Lee Jae-Hyuk Lee

Departments of Pathology, ${ }^{1}$ Forensic Medicine, and ${ }^{2}$ Neurology, Chonnam National University Medical School, Hwasun, Korea

Received: February 14, 2019

Revised: March 12, 2019

Accepted: March 14, 2019

\section{Corresponding Author}

Kyung-Hwa Lee, MD, PhD

Department of Pathology, Chonnam National University Medical School, 264 Seoyang-ro, Hwasun 58128, Korea

Tel: +82-61-379-7050

Fax: +82-61-379-7099

E-mail:mkkaylee@jnu.ac.kr

Jae-Hyuk Lee, MD, PhD

Department of Pathology,

Chonnam National University Medical School, 264 Seoyang-ro, Hwasun 58128, Korea

Tel: +82-61-379-7073

Fax: +82-61-379-7099

E-mail: jhlee@chonnam.ac.kr
Due to the progressive aging of Korean society and the introduction of brain banks to the Korean medical system, the possibility that pathologists will have access to healthy elderly brains has increased. The histopathological analysis of an elderly brain from a subject with relatively well-preserved cognition is quite different from that of a brain from a demented subject. Additionally, the histology of elderly brains differs from that of young brains. This brief review discusses primary age-related tauopathy; this term was coined to describe elderly brains with Alzheimer's diseasetype neurofibrillary tangles mainly confined to medial temporal structures, and no $\beta$-amyloid pathology.

Key Words: Autopsy; Cognition; Dementia; Tauopathies; Amyloid beta-peptides
Even before the term "primary age-related tauopathy (PART)" was proposed in 2014, pathologists had observed localized neurofibrillary degeneration in brains from aged people with relatively well-preserved cognitive function that was mostly restricted to medial temporal regions. These findings were somewhat informally described as "aging changes" because the features were considered insufficient for a diagnosis of Alzheimer disease (AD). The newly proposed consensus term (i.e., PART) includes features that range from the presence of isolated neurofibrillary tangles (NFTs) in cognitively normal aged brains to a subtype of frontotemporal lobe degeneration (FTLD) known as FTLD-tau, which is also referred to as tangle-only dementia, tangle-predominant senile dementia (TPSD), and preferential development of NFT without senile plaques. ${ }^{1,2}$ However, these previous designations tended to accentuate unnecessarily the clinical aspects of cognitive impairment, leading to a biased understanding of the disease entity. ${ }^{1}$ Although the word dementia is included in these terms, profound cognitive impairment that interferes with daily activities occurs in only a minority of affected individuals within this population. ${ }^{1}$

Thus, the consensus term PART was suggested by researchers of neurodegenerative diseases as a more objective and quantitative description of pathological disease status separate from the clinical presentation. The term PART was inspired by the pathological classification system for AD of the National Institute on AgingAlzheimer's Association. ${ }^{1,3}$ Since being introduced, the clinicopathological traits of PART have been clarified more precisely. ${ }^{4-8}$ This review aims to increase recognition of this disease entity by Korean pathologists through a literature review and discussion of the clinicopathological implications of PART, and via a figu- 
rative presentation of a PART case recently diagnosed at Chonnam National University Hospital brain bank.

\section{DEFINITION AND HISTOLOGICAL SPECTRUM OF PRIMARY AGE-RELATED TAUOPATHY}

The diagnosis of PART is histological in nature and can be applied to patients exhibiting mild-to-moderate tau-positive NFTs but without, or with few, $\beta$-amyloid $(A \beta)$ plaques. ${ }^{1,9}$ The gross features of a brain with PART may include minimal atrophy that is primarily located in the medial temporal lobe; however, diffuse neocortical atrophy is also present in some cases. AD-type NFTs, including ghost tangles, are mainly distributed in the hippocampus and medial temporal lobe (Fig. 1A, in comparison with advanced AD, Fig. 1B), and correspond to Braak stages I-III in the majority of patients and to stage IV in rare cases. ${ }^{9}$ The neuronal tauopathy of PART may also extend to granule cells of the dentate gyrus and neurons in the CA4 subregion of the hippocampus (Fig. 1C). ${ }^{9}$ Other than PART, the presence of ghost tangles or tau involvement in the dentate gyrus and CA4 are typically considered to be features of advanced AD (Fig. 1D). ${ }^{9}$ In addition to the hippocampus and medial temporal lobe, NFTs may also be observed in subcortical structures, such as the amygdala, nucleus basalis of Meynert, nucleus accumbens, hypothalamus, thalamus, and olfactory system (bulb and cortex), and in the brainstem, including the substantia nigra, locus coeruleus, dorsal raphe nucleus, and medulla oblongata, where NFTs develop at a younger age, sometimes even in teenagers. ${ }^{1,10,11}$ Biochemical and immunohistochemical studies have revealed that the NFTs in PART contain mixed three-repeat (3R) and four-repeat (4R) isoforms of tau proteins, as seen in $\mathrm{AD}{ }^{1,9}$

The presence of NFTs in Braak stage IV or lower is a basic requirement for a histological diagnosis of PART; when combined with Thal $A \beta$ phase 0 , PART can be diagnosed definitively (Fig. $1 \mathrm{E}-\mathrm{G})$. If the required distribution of NFTs is observed together with Thal $A \beta$ phase 1 or 2 , then the pathology is categorized as possible PART. ${ }^{1}$ The following is an example pathological diagnosis of PART: "Primary age-related tauopathy (PART), Definite, Braak stage III." Although Braak stage IV is considered a requirement for PART diagnosis, Braak stage IV pathology in the absence of $A \beta$ plaques is rare and the possibility that cases such as these represent FTLD-tau needs to be considered. ${ }^{1}$

\section{GENETIC AND CLINICAL ASPECTS OF PART}

PART brains are deficient in the apolipoprotein $\mathrm{E}$ (APOE) $\varepsilon 4$ allele, which is highly associated with the risk of $\mathrm{AD}^{2}{ }^{2}$ The frequency of APOE $\varepsilon 4$ in PART is approximately $10 \%,{ }^{1,6}$ whereas its prevalence in $\mathrm{AD}$ exceeds $45 \% \cdot{ }^{12,13} \mathrm{~A}$ major genetic risk factor for PART is the microtubule-associated protein tau (MAPT) gene $\mathrm{H} 1$ haplotype, which is also an accepted risk factor of progressive supranuclear palsy (PSP), corticobasal degeneration (CBD), and argyrophilic grain disease (AGD)., ${ }^{2,14}$

By definition, patients with the PART pathology present with a lack of, or minimal, cognitive impairment. ${ }^{2}$ However, greater impairment has been noted as an aspect of TPSD, where the initial symptoms typically include memory disturbances. ${ }^{15}$ During disease progression, deficits in cognitive function may extend to mild cognitive impairment (MCI) with a relatively well-preserved personality. ${ }^{15}$ Mental derangements, such as disorientation (or rarely, delirium), depression and paranoid thinking have also been observed. ${ }^{15,16}$ One feature of PART is that cases with higher NFT stages (Braak stage III or IV) are more likely to be associated with subjective memory impairments, which is a common complaint among the elderly population. ${ }^{17,18}$

\section{DEBATE REGARDING WHETHER PART IS ON THE ALZHEIMER DISEASE CONTINUUM}

Considering PART as being on the AD continuum, and especially comparing it with the limbic-predominant form of $\mathrm{AD}$, has been discouraged for several reasons. ${ }^{6,916,19}$ First, PART is associated with lower Braak NFT stages and fewer, or an absence of, $A \beta$ plaques. Second, patients with PART have an older age of onset, shorter disease duration, and less severe cognitive impairment. Third, the frequency of APOE $\& 4$ is much lower in PART than in the normal elderly population and the frequency of TDP43 proteinopathy is higher in patients with limbic-predominant $\operatorname{AD}(67 \%)$ than in those with definite PART (29\%) (Table 1). ${ }^{20}$

A discussion on the role that $A \beta$ plays in tauopathy is inevitable when PART is compared with $A D$. In the absence of $A \beta$, as seen in definite PART, the severity of tau-positive NFTs tends to be greater with older age at death. ${ }^{6}$ A proposed pathological step in late-onset $\mathrm{AD}$ is tauopathy, corresponding to PART, which is purported to occur at some point in the life cycle of almost every individual; amyloidosis may also occur as an independent event. ${ }^{21}$ In this model, $A \beta$ is not a catalyst for tau deposition in the brain, but rather serves to promote the spread of tauopathy. This hypothesis is supported by a study that used an established cell biosensor 
PART
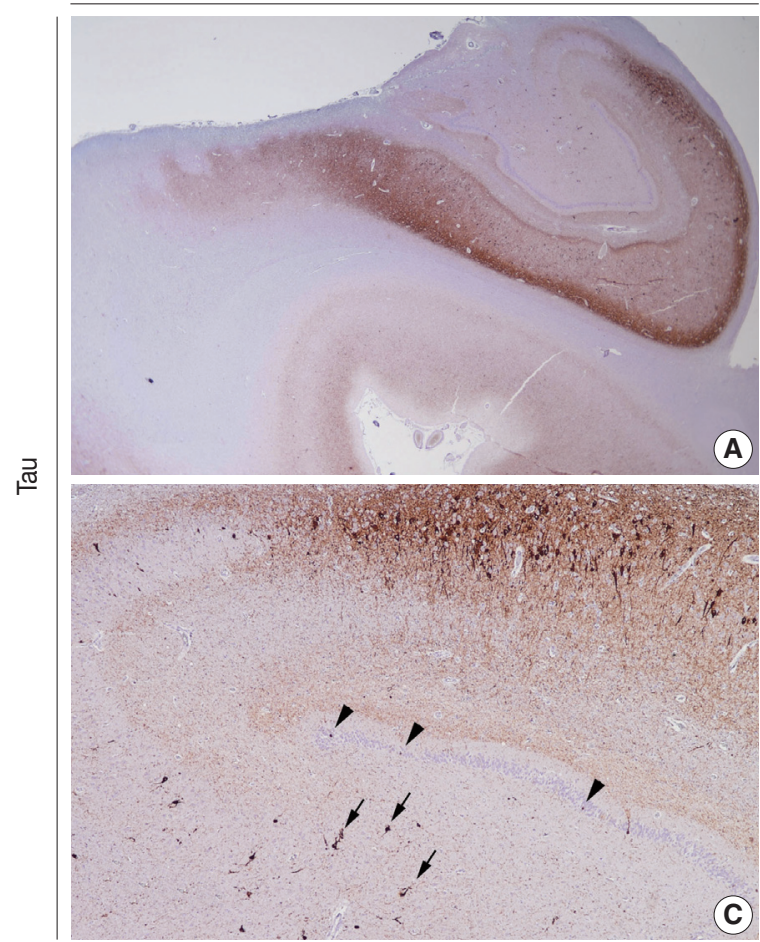

(C)

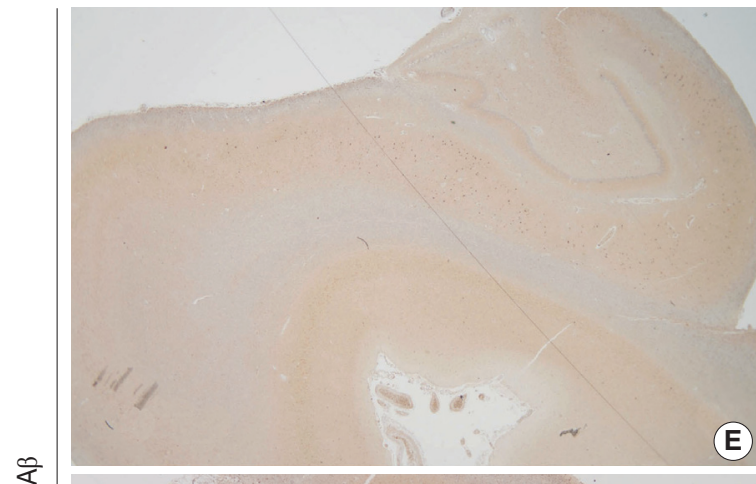

\&

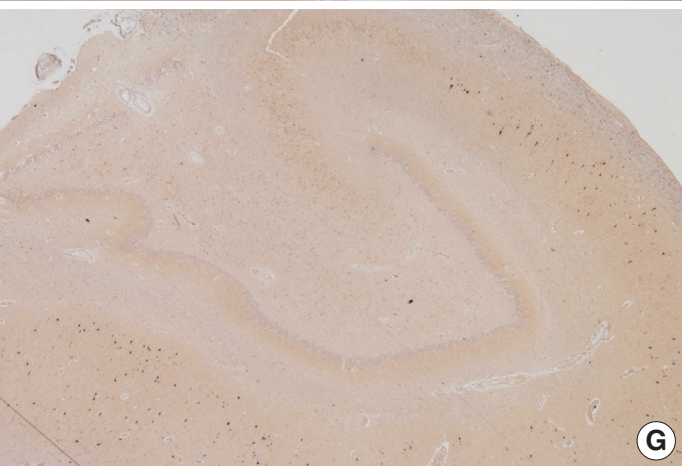

High ADNC
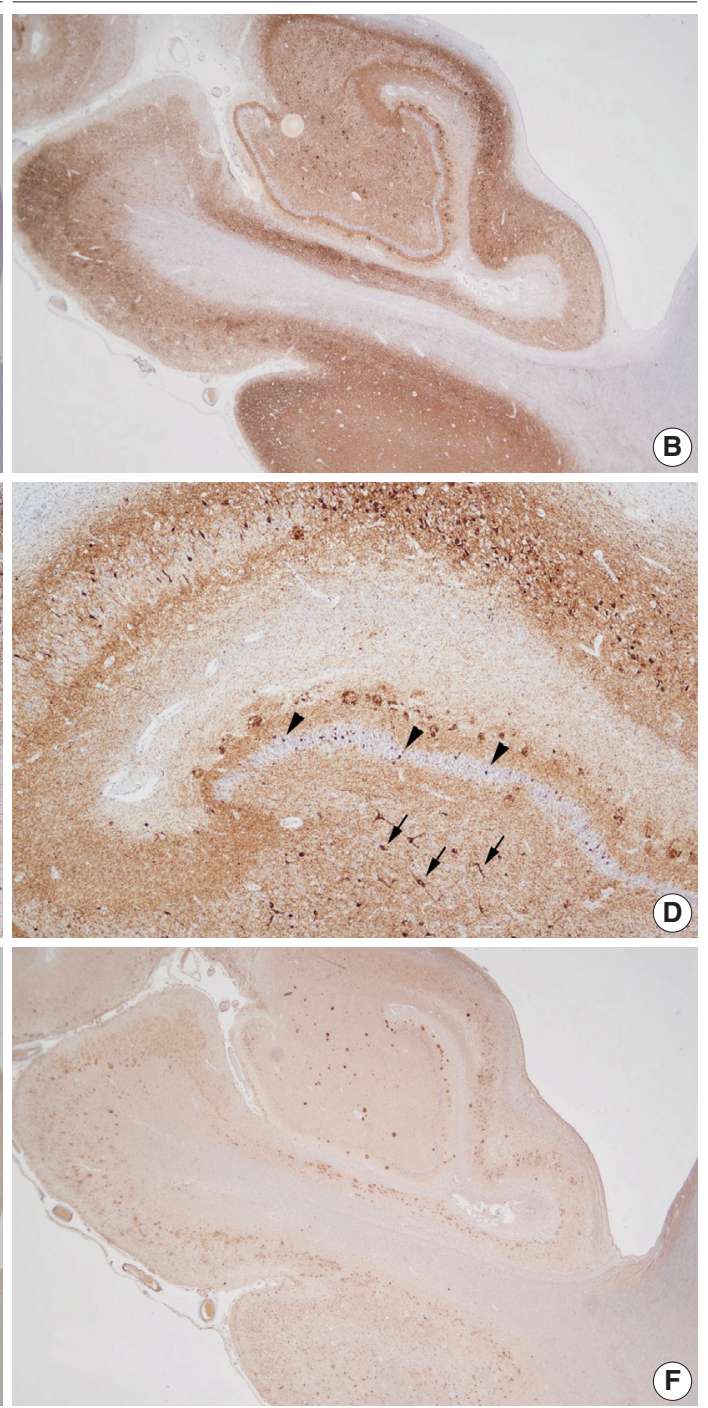

(

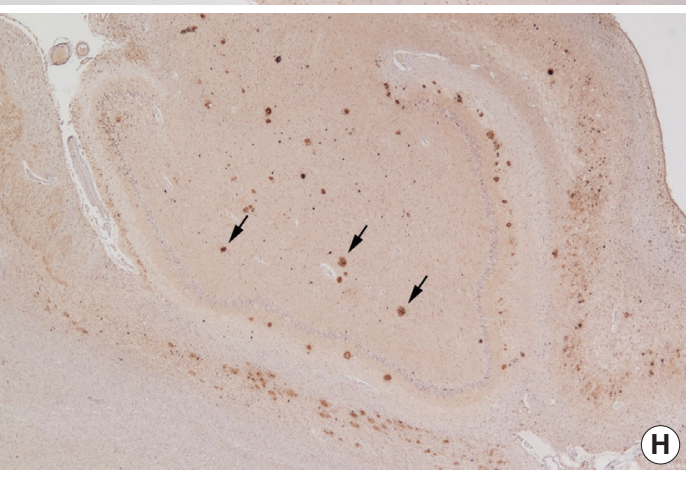

Fig. 1. Histopathological findings of primary age-related tauopathy (PART) in a 92-year-old woman (A, C, E, G) compared with those of highAlzheimer's disease (AD) neuropathologic change (ADNC) in an 82-year-old man (B, D, F, H). Tau immunohistochemistry analyses revealed marked tauopathy centered in the hippocampus and subiculum of the PART brain (A) and the extension of tauopathy into the temporal neocortex of the high-ADNC brain (B) (AT8 immunohistochemistry). At a higher magnification, the granule cells of the dentate gyrus (arrowheads) and the neurons of the CA4 subregion (arrows) exhibited tau involvement in the PART brain (C), as well as in the brain of the advanced AD case (D) (AT8 immunohistochemistry). Although the hippocampus in the PART brain did not reveal any $\beta$-amyloid (AB)-positive plaques (E, G) ( $A \beta$ immunohistochemistry), the high ADNC brain showed A $\beta$ deposition in the temporal neocortex through the CA4 subregion (arrows) that corresponded to Thal $A \beta$ phase 4 (F, H) (Aß immunohistochemistry). 
Table 1. Comparison of PART with $A D$

\begin{tabular}{|c|c|c|}
\hline Variable & PART & $A D$ \\
\hline \multicolumn{3}{|l|}{ Primary pathology } \\
\hline Tau-positive NFTs & $\begin{array}{l}\text { Medial temporal } \\
\text { region-restricted } \\
\text { Braak stage } \leq \text { IV } \\
\text { (usually I-III) }\end{array}$ & $\begin{array}{l}\text { Diffuse cortical } \\
\text { distribution } \\
\text { Braak stage } \geq I I I \\
\text { (usually IV-VI) }\end{array}$ \\
\hline$A \beta$ plaques & $\begin{array}{l}\text { Thal phase 0-2 } \\
\text { Definite PART: } \\
\text { Thal phase 0 } \\
\text { Possible PART: } \\
\text { Thal phase 1-2 }\end{array}$ & Thal phase $\geq 3$ \\
\hline Tau isoforms & Mixed $3 \mathrm{R}$ and $4 \mathrm{R}$ tau & Mixed 3R and 4R tau \\
\hline Genetic association & MAPT H1 haplotype & APOE $\varepsilon 4$ \\
\hline \multicolumn{3}{|l|}{ Clinical features } \\
\hline Cognition & $\begin{array}{l}\text { Normal-to-mild } \\
\text { impairment }\end{array}$ & Dementia \\
\hline Duration (yr) & 5 & $9^{16}$ \\
\hline Age at death (yr) & 86 & $79^{14,19}$ \\
\hline Prevalence at $\geq 80 \mathrm{yr}(\%)$ & 20 & $80^{8,11}$ \\
\hline \multicolumn{3}{|l|}{ Other co-existing pathology } \\
\hline TDP-43 proteinopathy (\%) & 30 & $67^{14,19,20}$ \\
\hline Hippocampal sclerosis (\%) & 10 & $3^{16,20}$ \\
\hline $\begin{array}{l}\alpha \text {-Synuclein positive Lewy } \\
\text { bodies (\%) }\end{array}$ & 10 & $30^{14,20}$ \\
\hline
\end{tabular}

PART, primary age-related tauopathy; AD, Alzheimer's disease; NFTs, neurofibrillary tangles; $A \beta, \beta$-amyloid; 3R, three-repeat; 4R, four-repeat.

assay to show that the seeding activity of tauopathy is enhanced in the presence of $A \beta$-positive plaques. ${ }^{4}$ However, another report found no clear distinction in tau seeding activity between PART and $\mathrm{AD}$ subjects. ${ }^{7}$ The functional interaction between tau and $A \beta$, as well as the relationship between PART and $A D$, remain to be elucidated. ${ }^{2,22}$

\section{COEXISTING PATHOLOGIES IN ELDERLY INDIVIDUALS WITH PRIMARY AGE-RELATED TAUOPATHY}

Previous studies focused on brain pathology in the elderly have identified several pathological trends. ${ }^{8,11,18}$ Roughly $20 \%$ of aged people in their 80 s or older have PART, ${ }^{8,11,18,23}$ while the remaining $80 \%$ exhibit some degree of AD-type pathology characterized by both NFTs and neuritic plaques. ${ }^{11}$ Moreover, there are few cases that are $A \beta$-positive only; most cases of $A \beta$ deposition show some tau-positive NFTs. ${ }^{8,11}$ These observations are consistent with prior reports showing that approximately $25 \%$ of elderly people with well-preserved cognitive function lack brain amyloidosis. ${ }^{1}$ Even among a carefully selected group of centenarians, $20 \%$ were relatively free of $A \beta$ deposition as detected by immunohistochemistry. ${ }^{11}$ Thus, the relative proportions of PART and
$\mathrm{AD}$ appear to be maintained in centenarian populations. ${ }^{8,11}$

It has been proposed that a neuropathological diagnosis of TPSD or PART should be conservatively applied to cases where NFTs mainly affect the hippocampal/limbic area, and where there is a scarcity of $A \beta$ deposits and no evidence of any other dementia characterized by NFTs. ${ }^{15}$ The limits of the PART diagnosis explain the exclusion of PSP, CBD, and even Lewy body disease cases in previous studies on PART. ${ }^{6}$ Despite the proposed limitations to its diagnostic criteria, PART has been shown to co-exist with other pathologies. For example, TDP-43 proteinopathy, including cerebral age-related TDP-43 with sclerosis, and AGD have been identified in approximately $30 \%$ of cases with definite PART. ${ }^{6}$ Similarly, hippocampal sclerosis has been identified in approximately $10 \%$ of cases ${ }^{16}$ while $\alpha$-synuclein-positive Lewy bodies have been observed in fewer than $10 \%$ of PART cases. ${ }^{6}$

\section{CONCLUSIONS}

PART can be diagnosed in cases showing neurofibrillary degeneration restricted to the medial temporal region, an absence (or scarcity) of $A \beta$ deposition, and a lack of cognitive impairment or MCI. However, several unanswered issues remain regarding the "gray zone" between PART and AD. The definition of clinicopathological PART requires refinement due to the presence of clinical PART in cases with higher Thal $A \beta$ phases and moderate-to-frequent neuritic plaques. ${ }^{18}$ The implications of the association of PART with diffuse amyloid and neuritic plaques also remain to be clarified, although a quantitative margin of $A \beta$ deposition, up to Thal phase 2, has been proposed. ${ }^{1}$ Neither an exact diagnostic threshold for $A \beta$ deposition nor the methodology with which to detect its presence has been clearly defined. Furthermore, the term "age-related" is somewhat ambiguous. For example, the accumulation of abnormally phosphorylated tau proteins can begin before puberty and Braak stage I or II may be seen in individuals in their 20s. ${ }^{11}$ Determining a common pathology in aged brains, with recognition of PART, will provide a firm foundation for a more profound understanding of age-related neurodegenerative changes. Additionally, accumulated neuropathology data reflecting the epidemiology of the Korean population will be a good resource for future neuroscientific research.

\section{ORCID}

Daru Kim: https://orcid.org/0000-0001-5382-1206

Hyung-Seok Kim: https://orcid.org/0000-0002-8297-9747

Seong-Min Choi: https://orcid.org/0000-0003-3138-1881 
Byeong C. Kim: https://orcid.org/0000-0001-6827-6730

Min-Cheol Lee: https://orcid.org/0000-0002-0799-2976

Kyung-Hwa Lee: https://orcid.org/0000-0002-3935-0361

Jae-Hyuk Le: https://orcid.org/0000-0002-8934-0145

\section{Author Contributions}

Conceptualization: MCL.

Funding acquisition: KHL.

Investigation: SMC.

Methodology: HSK, DK.

Project administration: JHL.

Supervision: JHL.

Validation: MCL, KHL.

Visualization: DK, KHL.

Writing—original draft: DK.

Writing—review \& editing: HSK, SMC, BCK, KHL.

\section{Conflicts of Interest}

The authors declare that they have no potential conflicts of interest.

\section{Acknowledgments}

This work was supported by a National Research Foundation of Korea (NRF) grant funded by the government of Korea (MSIT; No. 2016R1A2B1014597, 2018R1A5A2024181).

\section{REFERENCES}

1. Crary JF, Trojanowski JQ, Schneider JA, et al. Primary age-related tauopathy (PART): a common pathology associated with human aging. Acta Neuropathol 2014; 128: 755-66.

2. Irwin DJ. Tauopathies as clinicopathological entities. Parkinsonism Relat Disord 2016; 22 Suppl 1: S29-33.

3. Hyman BT, Phelps CH, Beach TG, et al. National Institute on AgingAlzheimer's Association guidelines for the neuropathologic assessment of Alzheimer's disease. Alzheimers Dement 2012; 8: 1-13.

4. Bennett RE, DeVos SL, Dujardin S, et al. Enhanced tau aggregation in the presence of amyloid beta. Am J Pathol 2017; 187: 1601-12.

5. Crary JF. Primary age-related tauopathy and the amyloid cascade hypothesis: the exception that proves the rule? J Neurol Neuromedicine 2016; 1: 53-7.

6. Josephs KA, Murray ME, Tosakulwong N, et al. Tau aggregation influences cognition and hippocampal atrophy in the absence of beta-amyloid: a clinico-imaging-pathological study of primary agerelated tauopathy (PART). Acta Neuropathol 2017; 133: 705-15.

7. Kaufman SK, Del Tredici K, Thomas TL, Braak H, Diamond MI. Tau seeding activity begins in the transentorhinal/entorhinal regions and anticipates phospho-tau pathology in Alzheimer's disease and PART. Acta Neuropathol 2018; 136: 57-67.

8. Neltner JH, Abner EL, Jicha GA, et al. Brain pathologies in extreme old age. Neurobiol Aging 2016; 37: 1-11.

9. Kovacs GG. Invited review: neuropathology of tauopathies: principles and practice. Neuropathol Appl Neurobiol 2015; 41: 3-23.

10. Braak H, Del Tredici K. The pathological process underlying Alzheimer's disease in individuals under thirty. Acta Neuropathol 2011; 121: 171-81.

11. Braak H, Thal DR, Ghebremedhin E, Del Tredici K. Stages of the pathologic process in Alzheimer disease: age categories from 1 to 100 years. J Neuropathol Exp Neurol 2011; 70: 960-9.

12. Jicha GA, Parisi JE, Dickson DW, et al. Age and apoE associations with complex pathologic features in Alzheimer's disease. J Neurol Sci 2008; 273: 34-9.

13. Alzheimer's Association. 2016 Alzheimer's disease facts and figures. Alzheimers Dement 2016; 12: 459-509.

14. Janocko NJ, Brodersen KA, Soto-Ortolaza AI, et al. Neuropathologically defined subtypes of Alzheimer's disease differ significantly from neurofibrillary tangle-predominant dementia. Acta Neuropathol 2012; 124: 681-92.

15. Yamada M. Senile dementia of the neurofibrillary tangle type (tangle-only dementia): neuropathological criteria and clinical guidelines for diagnosis. Neuropathology 2003; 23: 311-7.

16. Jellinger KA, Attems J. Neurofibrillary tangle-predominant dementia: comparison with classical Alzheimer disease. Acta Neuropathol 2007; 113: 107-17.

17. Kryscio RJ, Abner EL, Jicha GA, et al. Self-reported memory complaints: a comparison of demented and unimpaired outcomes. J Prev Alzheimers Dis 2016; 3: 13-9.

18. Nelson PT, Trojanowski JQ, Abner EL, et al. "New old pathologies": AD, PART, and cerebral age-related TDP-43 with sclerosis (CARTS). J Neuropathol Exp Neurol 2016; 75: 482-98.

19. Murray ME, Graff-Radford NR, Ross OA, Petersen RC, Duara R, Dickson DW. Neuropathologically defined subtypes of Alzheimer's disease with distinct clinical characteristics: a retrospective study. Lancet Neurol 2011; 10: 785-96.

20. Josephs KA, Whitwell JL, Tosakulwong N, et al. TAR DNA-binding protein 43 and pathological subtype of Alzheimer's disease impact clinical features. Ann Neurol 2015; 78: 697-709.

21. Jack CR Jr. PART and SNAP. Acta Neuropathol 2014; 128: 773-6.

22. Duyckaerts C, Braak H, Brion JP, et al. PART is part of Alzheimer disease. Acta Neuropathol 2015; 129: 749-56.

23. Robinson JL, Corrada MM, Kovacs GG, et al. Non-Alzheimer's contributions to dementia and cognitive resilience in The 90+ Study. Acta Neuropathol 2018; 136: 377-88. 